\title{
Terrorism and Public Health: The Little Known Equation
}

\author{
Ron St.John ${ }^{1}$ \\ Claude Giroux ${ }^{2}$
} S itting in our offices early in the morning of September 11, 2001, we, like many across the world, watched with incredulity the twin terrorist strikes on the World Trade Centre. Riveted to the television screen, we witnessed the beginnings of a nightmare whose images will likely remain with us for a long time. September 11, and the events that followed, changed our assumptions of world terrorism and, at the same time, redefined public vulnerabilities to weapons of mass destruction using chemical, biological or radionuclear agents (CBRN) to disrupt social infrastructures and institutions by threatening life and limb as well as our sense of security.

Not for several years was there a beginning comprehension that the consequences of use of a biological weapon would be an epidemic and that those first detecting its presence and those primarily responsible for controlling the disease would be public health personnel and physicians. ${ }^{1}$

One of the key lessons of the September 11 terrorists attacks is that although a government can invest significantly in deterrence, attacks will still happen with devastating effects. While it is clearly important to invest in deterrence measures (policy and security) to counter terrorist activities, the question must be asked if we are investing strategically to achieve a balance between national security and public health security or, said differently, between deterrence and consequence management of disasters and emergencies.

The assessment of terrorist threats shows that Canada is as vulnerable as any industrialized nation, and maybe more so, given our proximity and close economic ties to the United States (US). Significant investment in preventing terrorism appears an obvious choice. Yet it may be that in focusing on national and continental security, we are neglecting an important aspect of terrorist actions: they occur inside national and continental security perimeters.

Citizens want to know that their governments can effectively deter terrorist actions. It is one of the basic roles of government to provide for the security of their citizens. Governments have heard the call and are investing significantly in the deterrence side of the equation of emergency preparedness and response. Threat assessment analysis indicates that the probability of a CBRN terrorist event is very low and yet we are expending limited public funds in preparing for emergencies that may never happen. Some would argue that we are investing disproportionately in deterrence strategies that may ultimately fail, especially when considering the potentially greater health and social gains that could be derived through a more balanced scheme that would enhance the system of and for public health.

One of the legacies of the terrorist attacks in the US is that North Americans are acutely aware that a society that depends on multiple complex systems is vulnerable to attacks that can disrupt its basic building blocks. In Canada, the immediate consequences of the US events and the closure of US airspace to all traffic except the military left some

Centre for Emergency Preparedness and Response, Population and Public Health Branch, Health Canada

1. Executive Director

2. Senior Policy Advisor

Acknowledgements: The authors wish to express their gratitude to Dr. Robert McMurtry, former Assistant Deputy Minister, Population and Public Health Branch, Health Canada, for reviewing the editorial and providing insightful suggestions.

The views expressed by the authors are theirs alone and do not necessarily reflect the views of Health Canada.
47,000 passengers stranded in Maritime airports and airports across Canada for close to five days. This posed an immediate challenge to provincial authorities to provide support and amenities to these reluctant visitors. The massive influx of these passengers on Canadian soil demonstrated that national borders provide no protection in a continental setting where terrorist actions using weapons of mass destruction occur. Given any event of similar proportions in the US, it is inevitable that the effects would be felt in Canada.

The single and maybe the most significant shift in thinking that has occurred since the terrorist attacks is the growing perception that the public health infrastructure is of greater importance than previously recognized. In truth, it is an essential and strategic component in the arsenal of measures to prepare for and respond to the threat of terrorist actions using CBRN agents and most especially for managing its consequences.

Any emergency evolves along a continuum of time and magnitude. The management of such emergencies involves four basic components: prevention (security and intelligence), detection (surveillance and diagnostic capacity), response (consequence management), and recovery. Public health authorities are involved in all aspects of emergencies management. Deterrence is a component of prevention while public health is chiefly concerned with detection, response and recovery.

It may come as a surprise to some to discover that public health professionals have always been involved in health security by the very nature of public health practices: detecting health hazards and risks through surveillance; risk and threat mitigation though promotion and prevention; managing the consequences of disease outbreaks; and assisting community efforts in recovering from disasters and emergencies. A public health emergency mobilizes similar resources regardless of the cause. The resources and expertise that are committed to detect, limit and manage health emergencies or bioterrorist actions are the same. For example, if terrorists were to infect segments of the Canadian population with the Ebola virus, the smallpox virus or the anthrax bacillus, the public health infrastructure would be on the front line using established practices and mobilizing the same surveillance, laboratory, medical 
expertise and support services. The difference between the two is that in the event of a terrorist attack, additional consideration must be given to the psychological impact and the provision of appropriate mental health and social support services.

At the outset of the anthrax hoaxes, the Health Canada Centre for Emergency Preparedness and Response initiated regular communications with Chief Medical Officers of Health and provincial and territorial emergency health services officials across the country and recommended that public health officials exercise a heightened index of suspicion. Public health authorities did what they always do, which is: monitor the health status of the population and identify health risks; diagnose and investigate health problems and hazards; mobilize community resources; inform, educate and empower communities and individuals to deal with health issues; develop policies, programs and plans supporting community health; employ innovative solutions to health problems; and work closely with other local and provincial authorities (fire, police) to manage the emergencies. This national effort would not have functioned effectively without the community-based public health organizations and the cooperation among disciplines working in the public health domain. Many of the initial gaps that would later be identified by the media had already been compensated for, owing to the expertise available in the existing public health networks and not insignificantly to the dedication of public health officials.

Public Health deals with an incredible array of issues that could and do affect the health of communities. One of its most effective tools in addressing health problems is actively weaving working partnerships with local authorities such as municipal administrations, fire departments and police services, voluntary associations, schools and the private sector (notably local pharmacies), to promote health and deal with health risks as they arise. The anthrax hoaxes reveal how effective and timely the community focus of public health authorities can be.
Is the conclusion here that we need not invest in the public health infrastructure since it managed to deal with the anthrax alerts? Far from it. The alerts demonstrated how vulnerable the all hazards response system is. Public health officials in many areas of the country were working flat out and would not have been able to sustain such a level of effort. Local resources were stretched to the limit and on occasion exceeded by the demand of dealing with suspicious packages. The experience revealed that the public health professionals and supporting infrastructure in Canada responded magnificently to the crisis. The experience also revealed the need to enhance, strengthen and modernize public health capacities across all levels of government. Public health officials from local health authorities to laboratory experts, public health nurses, mental health professionals, social workers and chief medical officers were faced with impossible demands and long hours in the first weeks of the anthrax hoaxes.

It is clear that investing in the public health infrastructure capacity to manage the social dynamics and the health consequences of CBRN terrorist actions would also increase the capacity to manage other public health emergencies. Terrorist actions are not the only source of public health risks. A strong public health infrastructure is a social necessity for managing the consequences of acts of terror, and all other hazards such as climate extremes, earthquakes, etc.

September 11 has taught us that law, order and good government must equate with increased efforts to deter terrorism and ensure the security and well-being of all nations. Domestic, continental and international realities necessitate a great commitment and effort to minimize the possibility of terrorist actions and to encourage conflict resolution through nonviolent means. It is hoped that the option of more peaceful and constructive political solutions will be found so that populations are not caught in the crossfire of intolerance, opposing ideologies, armed conflict and terrorism.
Terrorism is a very real concern that must be decisively addressed, not in isolation but as part of addressing all other hazards that constitute a risk to the health of individuals, communities and nations. A balanced approach to social investments designed to protect and enhance security must be balanced with investments in an "all hazards" health security approach for emergency preparedness and response to protect the health of Canadians. Social policy and related investments need to be predicated on immediate as well as emerging risk factors, such as climate change and its consequences, which may prove to be the greater public health challenge. With climate change, we may witness in the near future the emergence of exotic diseases such as malaria. Strategic investments in public health infrastructures would ensure a more robust, sustainable and coordinated response to disease epidemics, whatever the cause may be.

In closing, we applaud all emergency preparedness and response professionals who have and continue to prepare for and respond to emergencies. The public health infrastructure is the "nerf de la guerre" (the essential or basic component to waging war or mounting a public health campaign without a strong public health infrastructure, detecting or managing the consequences of a CBRN terrorist action will not be successful and the cost in terms of lives lost that could have been saved will be high). There is an urgent need to strengthen its capacity to deal with all health emergencies. We must collectively ensure a strong capacity in policing and security, and support a balanced, flexible and effective emergency preparedness and response system.

\section{REFERENCE/REFÉRENCE}

1. Henderson DA. Congressional Testimonies. Hearing on the Threat of Bioterrorism and the Spread of Infectious Diseases. September 5 , 2001. (reproduced on the Association of Schools of Public Health website http://www.asph.org/ aa_section.cfm/3) 


\title{
Terrorisme et santé publique : l'équation mal connue
}

\author{
Ron St.John ${ }^{1}$ \\ Claude Giroux ${ }^{2}$
}

A ssis dans notre bureau tôt le matin du 11 septembre 2001, comme tant d'autres personnes partout dans le monde, nous regardions, incrédules, les attaques terroristes jumelles contre le World Trade Center. Rivés à l'écran de télévision, nous avons été témoins des débuts d'un cauchemar dont les images resteront longtemps imprimées dans nos esprits. Le 11 septembre et ses séquelles ont changé ce que nous prenions pour acquis au sujet du terrorisme mondial et nous ont forcés à redéfinir notre vulnérabilité par rapport aux armes de destruction massive qui utilisent des agents chimiques, biologiques ou radionucléaires (CBRN) pour désorganiser les infrastructures et les institutions sociales en portant atteinte à la vie, à l'intégrité physique et au sentiment de sécurité de la population.

[Traduction] Il a fallu plusieurs années pour que l'on commence à comprendre que l'utilisation d'une arme biologique entraînerait une épidémie, et que les premiers à détecter sa présence et les principaux responsables de la lutte contre la maladie seraient le personnel et les médecins de la santé publique. ${ }^{1}$

Nous avons tiré une grande leçon des attaques terroristes du 11 septembre : même lorsqu'un gouvernement investit des sommes considérables dans la dissuasion, cela n'empêche pas les attaques et leurs effets dévastateurs. Il faut bien sûr investir dans la dissuasion (les politiques et les mesures de sécurité) pour combattre le terrorisme, mais il faut aussi nous demander si nous investissons de façon stratégique, pour atteindre un juste équilibre entre la sécurité nationale et la protection de la santé publique, autrement dit entre la dissuasion et la gestion des conséquences des sinistres et des urgences.

L'évaluation de la menace terroriste montre que le Canada est tout aussi vulnérable que les autres nations industrialisées et peut-être même davantage, en raison de sa proximité et de ses liens économiques étroits avec les États-Unis. Des investissements massifs dans la prévention du terrorisme semblent aller de soi. Cependant, si nous nous concentrons exclusivement sur la sécurité du pays et du continent, nous négligerons un aspect important des actes terroristes : ils se produisent à l'intérieur des périmètres de sécurité nationaux et continentaux.

Les citoyens veulent être convaincus que leurs gouvernements peuvent efficacement prévenir les actes de terrorisme. L'un des rôles fondamentaux d'un gouvernement est d'assurer la sécurité de ses citoyens. Les gouvernements ont entendu l'appel et investissent beaucoup dans la dissuasion, qui n'est cependant que l'un des membres de l'équation des mesures et interventions d'urgence. L'analyse de la menace montre que la probabilité d'un acte terroriste de type CBRN est très faible, mais que nous dépensons des fonds publics limités à nous préparer pour des urgences qui pourraient ne jamais se manifester. Certains prétendent que nous investissons des sommes disproportionnées dans des stratégies de dissuasion vouées à l'échec, surtout si l'on considère les gains sanitaires et sociaux supérieurs que l'on pourrait tirer d'un plan équilibré d'amélioration du système de santé publique.

Centre de mesures et d'interventions d'urgences, Direction générale de la santé de la population et de la santé publique, Santé Canada

1. Directeur administratif

2. Conseiller supérieur en politiques

Remerciements : les auteurs remercient Robert McMurtry, ancien sous-ministre adjoint à la Direction générale de la santé de la population et de la santé publique de Santé Canada, qui a eu I'amabilité de réviser cet éditorial et de formuler des suggestions éclairées.

Les points de vue exprimés par les auteurs n'appartiennent qu'à eux et ne reflètent pas nécessairement l'opinion de Santé Canada.

MAY - JUNE 2002

Dans la foulée des attaques terroristes aux États-Unis, nous les Nord-Américains sommes maintenant douloureusement sensibles au fait qu'une société qui dépend de multiples systèmes complexes est vulnérable aux attaques qui peuvent désorganiser ses composantes de base. Au Canada, les attaques, puis la fermeture de l'espace aérien américain à tous les aéronefs sauf ceux de l'armée ont eu pour conséquence immédiate de coincer 47000 passagers dans les aéroports des Maritimes et du reste $\mathrm{du}$ Canada pendant près de cinq jours. Avant toute autre chose, les autorités provinciales ont dû offrir du soutien à ces visiteurs malgré eux et leur trouver des locaux. L'afflux de passagers en sol canadien a prouvé que les frontières nationales n'offrent aucune protection, à l'échelle continentale, lorsque des terroristes ont recours à des armes de destruction massive. À l'avenir, tout événement de proportion semblable aux États-Unis aura inévitablement des effets qui se feront sentir au Canada.

Ce qui a surtout changé dans notre façon de penser depuis les attaques terroristes, c'est que l'on s'est mis à accorder aux infrastructures de santé publique une importance nouvelle. En vérité, ces infrastructures sont un outil essentiel et stratégique de l'arsenal des mesures de préparation et de réponse à la menace d'actes terroristes à l'aide d'agents CBRN, et surtout à la gestion de leurs répercussions.

Toute urgence se situe le long d'un axe temporel et quantitatif. La gestion des urgences comporte quatre grands volets : la prévention (sécurité et renseignement), la détection (surveillance et capacité de diagnostic), la réponse (gestion des répercussions) et le rétablissement. Les autorités de santé publique participent à ces quatre aspects. La dissuasion est un élément de la prévention, tandis que la santé publique se préoccupe surtout de la détection, de la réponse et du rétablissement.

Certains seront étonnés d'apprendre que les professionnels de la santé publique ont toujours eu un rôle à jouer dans la protection de la santé, de par la nature même des méthodes de santé publique : détection des dangers et des risques pour la santé par la surveillance; atténuation des risques et des menaces par la promotion et la prévention; gestion des répercussions des éclosions de maladies; et aide aux efforts communautaires de rétablissement après un sinistre ou 
une urgence. Une urgence de santé publique mobilise des ressources semblables, quelles qu'en soient les causes. Les ressources et les compétences engagées pour détecter, restreindre et gérer une urgence sanitaire ou un acte de bioterrorisme sont les mêmes. Si des terroristes, par exemple, infectaient des segments de la population canadienne avec le virus d'Ebola ou de la variole, ou le bacille du charbon, les infrastructures de santé publique seraient aux premières lignes, à utiliser des méthodes établies et à mobiliser les mêmes services de surveillance, de laboratoire, de savoir-faire médical et de soutien. La différence, c'est qu'en cas d'attaque terroriste, il faudrait tenir compte des retombées psychologiques et fournir des services de santé mentale et de soutien social appropriés.

Au début des fausses alertes au bacille du charbon, le Centre de mesures et d'interventions d'urgence de Santé Canada a établi des communications périodiques avec les médecins hygiénistes en chef et les représentants des services de santé d'urgence provinciaux et territoriaux du pays et recommandé aux autorités de santé publique d'être davantage sur leurs gardes. Ces autorités ont fait ce qu'elles font toujours, c'est-à-dire suivre l'état de santé de la population et déceler les risques sanitaires; diagnostiquer et étudier les problèmes et les dangers pour la santé; mobiliser les ressources communautaires; informer, sensibiliser et habiliter les collectivités et les particuliers pour leur permettre de composer avec les questions de santé; élaborer des politiques, des programmes et des plans à l'appui de la santé communautaire; appliquer des solutions novatrices aux problèmes de santé; et travailler en étroite collaboration avec les autres autorités locales et provinciales (les services d'incendie et de police) pour gérer les urgences. Cet effort national n'aurait pas fonctionné efficacement sans les organismes communautaires de santé publique et la coopération entre les diverses disciplines du domaine de la santé publique. Avant même que les médias les signalent, bon nombre de lacunes avaient déjà été compensées grâce aux compétences disponibles dans les réseaux de santé publique existants, sans oublier le dévouement des autorités de santé publique.

La santé publique recouvre une foule d'enjeux susceptibles d'affecter la santé des collectivités. L'un de ses instruments les plus efficaces consiste à travailler main dans la main avec les autorités locales, comme les administrations municipales, les services d'incendie et de police, les associations bénévoles, les écoles et le secteur privé (plus particulièrement les pharmacies locales) afin de promouvoir la santé et de composer avec les risques sanitaires qui se manifestent. Les fausses alertes au bacille du charbon ont montré à quel point l'approche communautaire des autorités de santé publique peut être efficace et opportune.

Faut-il en conclure qu'il n'est pas nécessaire d'investir dans les infrastructures de santé publique, qui ont si bien répondu aux alertes au bacille du charbon? Bien au contraire. Ces alertes ont mis au jour la vulnérabilité du système de réaction "tous risques ». Dans bien des régions du pays, les autorités de santé publique ont travaillé jour et nuit et n'auraient pas pu soutenir un tel niveau d'effort. Les ressources locales ont été mises à rude épreuve et parfois dépassées par la nécessité de vérifier tous les colis suspects. L'expérience a révélé que les professionnels de la santé publique et les infrastructures sous-jacentes, au Canada, ont traversé la crise avec brio. Mais elle a aussi révélé qu'il fallait rehausser, renforcer et moderniser les capacités de la santé publique à tous les échelons du gouvernement. Les autorités de santé publique, des autorités sanitaires locales aux laborantins en passant par les infirmières de la santé publique, les professionnels de la santé mentale, les travailleurs sociaux et les médecins hygiénistes en chef, ont dû composer avec des exigences impossibles et travailler de longues heures les premières semaines des fausses alertes au bacille du charbon.

Il est clair qu'en investissant dans les infrastructures de santé publique pour accroître leur capacité de gérer la dynamique sociale et les répercussions sanitaires d'actes terroristes de type CBRN, on augmentera notre capacité de gérer les autres urgences de santé publique. Les risques pour la santé publique ne se résument pas aux seuls actes terroristes. Il faut impérativement avoir de solides infrastructures de santé publique pour que la société puisse gérer les conséquences des actes de terreur et tous les autres risques (températures extrêmes, tremblements de terre, etc.).

Le 11 septembre nous a appris que la loi, l'ordre et le bon gouvernement nécessitent un effort accru pour prévenir le terrorisme et assurer la sécurité et le bien-être de toutes les nations. Les réalités nationales, continentales et internationales imposent un engagement et des efforts importants pour réduire la possibilité d'actes terroristes et favoriser la résolution des conflits par des moyens non violents. Il faut espérer que l'on trouvera des solutions politiques pacifiques et constructives pour éviter que des populations ne se retrouvent coincées entre l'intolérance, les idéologies contraires, les conflits armés et le terrorisme.

Le terrorisme est une préoccupation très réelle qu'il faut aborder de façon décisive, non pas isolément, mais en tenant compte de tous les autres facteurs qui posent un risque pour la santé des particuliers, des collectivités et des nations. Il faut atteindre un équilibre entre les investissements sociaux, qui visent à protéger et à améliorer la sécurité, et les investissements dans les mesures et interventions d'urgence " tous risques", qui protègent la santé des Canadiens contre tous les types de dangers. Les investissements dans les politiques sociales et les aspects connexes doivent reposer sur les facteurs de risque présents et futurs, comme le changement climatique et ses répercussions, qui pourraient poser un défi de santé publique supérieur au terrorisme. Avec le changement climatique, nous pourrions assister très bientôt à l'émergence de maladies exotiques comme la malaria. Par un investissement stratégique dans nos infrastructures de santé publique, nous nous assurerons d'avoir une réponse vigoureuse, durable et coordonnée aux épidémies, quelles qu'en soient les causes.

Pour terminer, nous applaudissons tous les professionnels des mesures et interventions d'urgence qui continuent à répondre aux urgences et à s'y préparer. Les infrastructures de santé publique sont le nerf de la guerre; sans elles, il sera impossible de détecter à temps les actes de terrorisme de type CBRN ou d'en gérer les répercussions, ce qui pourrait coûter de nombreuses vies qui autrement auraient été épargnées. Il existe un besoin pressant de renforcer la capacité de composer avec toutes les urgences sanitaires. Ensemble, nous devons nous doter d'une forte capacité policière et de sécurité, tout en soutenant un système de mesures et interventions d'urgence à la fois équilibré, souple et efficace. 